\section{Full attention}

Attention and Effort. By Daniel Kahneman. Pp. $x+246$. (Series in Experimental Psychology.) (Prentice-Hall: Englewood Cliffs, N.J., July 1973.) $\$ 8.95$.

BETwEen the wars the topic of attenion was neglected by psychologists, in their enthusiasm for predicting each response as a function of the immediately previous stimulus. The difficulty was, however, that the effects of a stimulus depend upon the state in which a man is when he receives it. Sometimes he reacts to it, will say verbally that he has noticed it, will remember it later; and sometimes none of these things will happen. The processes which can be variously and roughly described as gating, filtering, or selecting among inputs to the system have therefore become a live area of research: and books on attention are now almost too numerous. They range from the massive, detailed, and unreadable to the popular, chatty and superficial. The competition for another is tough.

It is a pleasure therefore to be able to declare this book the best one on the subject thus far. It is short, clear, and extremely up to date: not merely are there references to publications in 1973 , but also exciting accounts of new experiments by the author and his students in Jerusalem, which will be carefully read by advanced workers in the field. At the same time, the coverage of the literature is wider than in most of the competitors, and the author understands sympathetically what is being argued by other investigators. Thus for once this is a book which can be recommended, not only to the advanced workers already mentioned, but for teaching purposes, and even conceivably for non-psychologists who wish to find out the latest state of the art in this area.

For those who are already expert, one can indicate the position the book adopts by saying that it takes broadly the position of Treisman, but with much greater emphasis and experimental detail on Neisser's pre-attentive processes, and on recursive influences from processes at one stage of recognition upon others both earlier and later in the system. The findings of the past two or three years make it possible to do this convincingly and briefly, and Fig. 5-1 is perhaps the first diagrammatic representation of attention which improves upon that of Treisman in 1960.

Criticisms? The book is weaker when it turns to motor performance rather than perception, which is reasonable because the state of knowledge is less satisfactory: and there are perfectly adequate references to Posner, to Keele, to Brown and to Trumbo and Noble for those who wish to go further. Those of us who work on the effects of noise and of other stresses will be discontented by the way in which effort, arousal, the allocation of resources and the process which determines the size of the pupil are all treated almost interchangeably. Perhaps one can most fairly put it this way: there are real differences, which experimental evidence compels us to draw, between easy and involuntary attention such as that with which we follow the graceful prose of this author; and the determined concentration, sustained by a hope of remote goals, with which we mobilise all our resources to follow the confused thinking of some others. These differences are not yet understood, but for what we know now, Kahneman is the best guide.

\section{Donald F. Broadbent}

\section{Nematode interactions}

Nematode Ecology and Plant Disease. By H. R. Wallace. Pp. ix +228. (Arnold: London, December 1973.) £6.50.

THIs is a readable and interesting account of nematode ecology and the role of nematodes in plant diseases. Farly chapters discuss nematode injury to plants, plant growth in relation to numbers of nematodes and plant responses to nematode invasion and feeding. The second half of the book deals with distribution, abundance and adaptations of plant-parasitic nematodes in the environment and with their involvement in specific diseases. The final chapter suggests how the role of nematodes in plant diseases should be investigated.

Unlike Dr Wallace's earlier book (Biology of plant-parasitic nematodes; 1963) this is no textbook, for it is far from comprehensive and the references are nearly all drawn from the last decade. The text is often speculative, not a bad thing, but it is also at times superficial, and with some conclusions that are debatable. The book is best where, as in parts of the chapters on nematode cology, it deak concisely with its subject. Too much of the text, in my opinion, is taken up with fulsome descriptions, definitions and debates on abstractions such as 'health', 'disease', 'homeostasis', and ccological jargon and with the views of medical and plant pathologists.

Too little attention is devoted to well documented evidence of the relationship of nematodes with plant diseases. For example, much is known of nematode-fungus interactions in plant disease complexes and of nematodes as vectors of plant viruses, both of which receive scant attention. The inferences drawn about the work on Docking disorder are incorrect. Nor does Wallace indicate how much can be learnt from careful study of plant symptoms and from inoculation experiments with suspected pathogens.

Instead, he stresses the use of multifactorial analyses of all the environmental variables that can be thought of and measured, to indicate which factor or combination of factors is most likely responsible for the particular disease. $\mathrm{T}_{0}$ my mind this is putting cart before horse. Not all diseases involving nematodes are as complex as Dr Wallace implies or we would be without commercially successful cultivars bred for resistance to specific nematodes. Despite such criticisms I commend this book to all thoughtful nematologists, plant pathologists and ecologists.

\section{A. G. Whitehead}

\section{Physicists talking}

Fundamental Interactions in Physics: 1973 Conference at the Centre for Mathematical Physics in the University of Miami. Edited by Arnold Perlmutter. Pp. ix +399. (Studies in Natural Sciences vol. 2.) (Plenum: London and New York, 1973.) \$29.

A CONFEREnce on so wide a subject as this one is in danger of losing coherence and producing a set of papers from so great a range of disciplines as to require from any one scientist an enormous effort of comprehension, and yet to include contributions so speculative as to make such cffort unworthwhile. This familiar danger is avoided by the present volume, largely because of the elegance and informality of the presentation of the papers which seem to have been presented as talks and recorded verbatim. Here, perhaps, is a solution to the problem of scientific style on which John Maddox commented in a recent $\mathrm{BBC}$ radio broadcast: ". . . when a scientist sits down in front of his IBM typewriter. . . ."

There are thrce sections in the book. The first deals with particle physics and is largely theoretical though it includes an informal description of work at the National Accelerator Laboratory (Batavia, Illinois) and a paper by Hofstadter on applications of absorption detectors. The theoretical papers range from a quasi-classical discussion by P. A. M. Dirac of symmetry breaking in a new variation of Weyl's theory of the electromagnetic field, to a terse and wholly algebraic "Approach to Hadron Physies . . ." by Oneda and Matsuda. Several of these papers contain much that is new, and some of them contain fertile ideas rather than completed theories. For example, Bopp and Lutzenberger in a short contribution throw out a simple model of quantised retarded forces in which the electron and the muon appear as eigensolutions sub- 